Review

\title{
Circular RNAs in Brain Disorders
}

\author{
Ruirui Jia ${ }^{1,2}$, Zhengguo Li 1,2, Chuan Huang 1,2,* \\ 1 School of Life Sciences, Chongqing University, Chongqing 401331, China \\ 2 Center of Plant Functional Genomics, Institute of Advanced Interdisciplinary \\ Studies, Chongqing University, Chongqing 401331, China \\ * Correspondence: Chuan Huang, Email: chuanhuang@cqu.edu.cn.
}

\begin{abstract}
Circular RNAs, a class of covalently closed transcripts, are generated from thousands of protein-coding genes or noncoding regions of the genome via non-canonical splicing, and serve as important controllers in a variety of biological events in eukaryotes. Emerging studies have shown that circular RNAs are enriched in animal brains, and involved in brain disorders. In this review, we will discuss recent progress on circular RNA biogenesis, nuclear export, stability control, molecular functions, and regulatory roles in brain functions.
\end{abstract}

KEYWORDS: circular RNA; brain disorder; clinical biomarker

\section{INTRODUCTION OF CIRCULAR RNA BIOLOGY: BIOGENESIS, NUCLEAR EXPORT, DEGRADATION, AND MOLECULAR FUNCTIONS OF CIRCULAR RNAS}

\section{Biogenesis}

Although a subset of circular RNAs are generated from noncoding regions, most of them are outputs of many protein-coding genes via backsplicing. In contrast to canonical splicing that joins the exons in a linear order (5' to 3 ' end), backsplicing joins the 3 ' end of exon to the 5 ' end of an upstream exon, and are typically controlled by various factors. (i) Flanking intronic repeats: in many cases, base pairing between the flanking intronic repeats of circularizing exons brings a splicing donor

\section{G Open Access}

Received: 29 August 2019

Accepted: 13 December 2019

Published: 16 December 2019

Copyright (C 2019 by the author(s). Licensee Hapres, London, United Kingdom. This is an open access article distributed under the terms and conditions of Creative Commons Attribution $\underline{4.0 \text { International License. }}$ and an upstream splicing acceptor in close proximity and facilitates circular RNA production (Figure 1A) [1-3]. There are many highly expressed circular RNAs are flanked by a pair of complementary repeats. Interestingly, due to the diverse landscape of complementary repeats, the conserved protein-coding genes produce different circular RNAs in different species. (ii) Protein factors: a subset of circular RNAs that are not flanked by complementary repeats are circularized by several RNA binding proteins. A well-known RNA binding factor-Quaking acts analogously as flanking repeats and facilitates circularization of hundreds of circular RNAs. During human epithelial-mesenchymal transition, Quaking directly binds to the upstream and downstream flanking intron of circularizing exons, resulting in close proximity of splicing sites (Figure 
1B) [4-7]. (iii) Exon skipping events: Using RNA-seq, Kelly et al. found that circularization of exons is correlated with exon skipping events globally in human umbilical vein endothelial cells, and Barrett et al. identified that mrps16 can generate a circular RNA via exon skipping in S. pombe (Figure 1C) $[8,9]$. (iv) Intron retention: although most introns are moved, a class of RNA polymerase II binding circular RNAs were reported to be circularized with introns "retained" between exons, and thus termed as exon-intron circular RNAs (EIciRNAs; Figure 1D) [10]. (v) Splicing machinery: Recent study found that circular RNAs become the preferred gene products when core spliceosomal components were depleted from cells or canonical mRNA splicing events were inhibited by Pladienolide B (Figure 1E), suggesting that the biogenesis of circular RNAs and their linear counterparts remain in a dynamic balance [11].

A

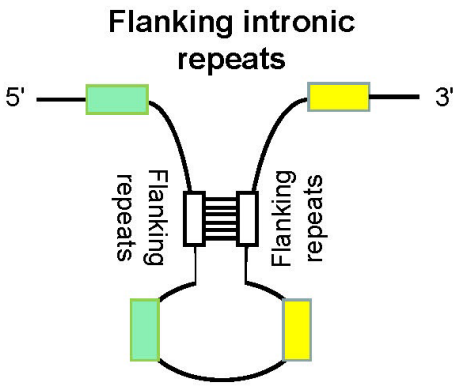

B Protein factors

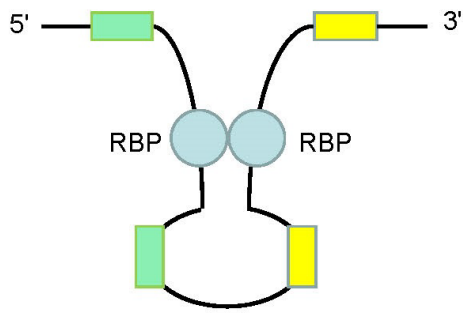

C

Exon skipping events

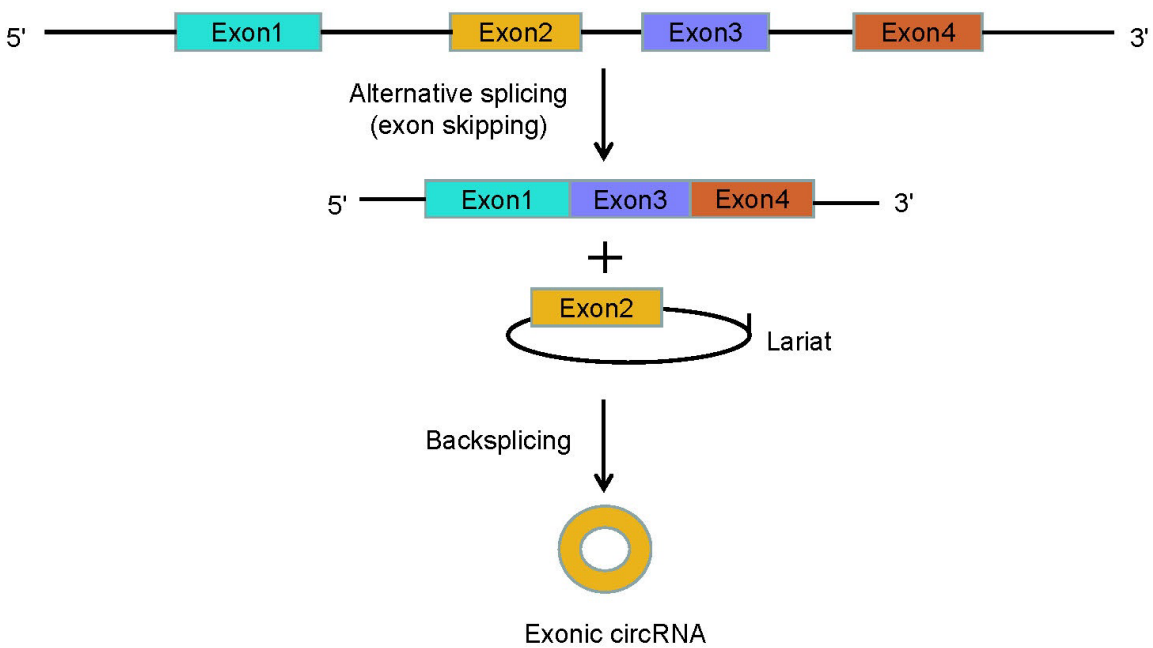

Figure 1. The biogenesis of circular RNAs. (A) Many circular RNAs trend to be flanked by intronic repeats. Base pairing between the flanking intronic repeats of circularizing exons facilitates backsplicing events that a splicing donor joins an upstream splicing acceptor. (B) In some cases, RNA-binding proteins (RBPs) act analogously as flanking repeats via direct interaction with flanking intron. (C) Circular RNA can also be generated by exon skipping events that result in a linear RNA and an exon-containing intron lariat, which is re-spliced to generate a mature exonic circular RNA. (D) Exon-intron circular RNAs are circularized with introns "retained" between exons. (E) The dynamic balance between linear and circular transcripts are controlled by spliceosome. Circular RNAs become the major gene products when core spliceosomal components were inhibited. 
D Intron retention

$5^{\prime}$
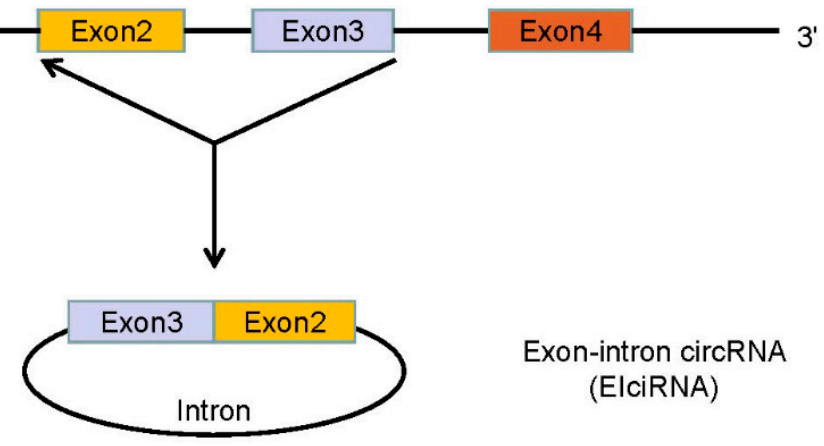

Exon-intron circRNA

(EIciRNA)

E

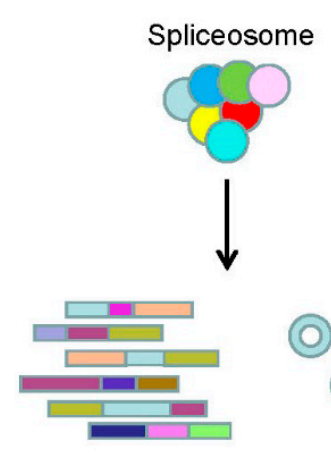

\section{Splicing machinery}

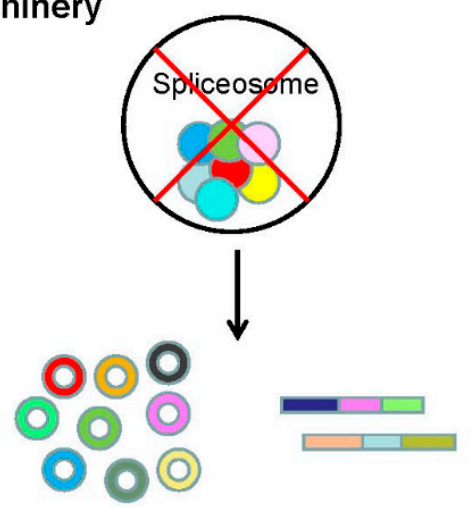

Figure 1. Cont.

\section{Nuclear Export}

Nuclear export of cytoplasmic circular RNAs is important to their proper functions. Although intronic or intron-containing circular RNAs are retained in the nucleus [10,12], most circular RNAs are efficiently exported to the cytoplasm [13,14]. Due to lack of canonical export signals, circular RNAs were thought to reach the cytoplasm upon nuclear envelope breakdown during cell division. However, recent studies proved that nuclear export of circular RNAs is an active process and regulated by conserved factors-UAP56 (DDX39A) and URH49 (DDX39B) in a sizedependent manner [15-17]. Long circular RNAs (>1298 nt) are exported by UAP56, while short circular RNAs (<356 nt) are exported by URH49 (Figure 2). Surprisingly, a four amino-acid region (KSLN in UAP56; RSFS in URH49) can determine circular RNA size preference. 


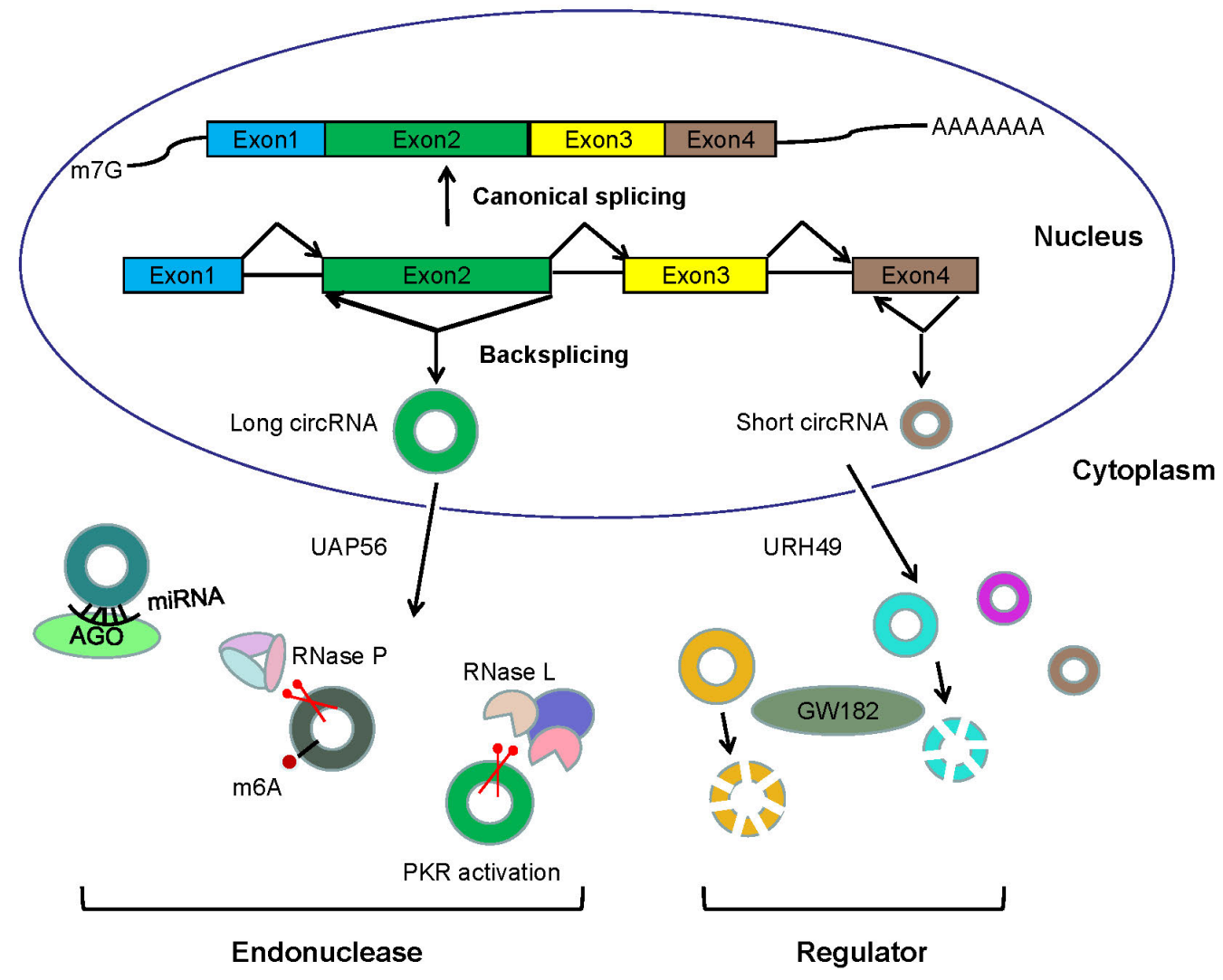

Figure 2. The nuclear export and degradation of circular RNAs. Circular RNAs are exported to cytoplasm in a size-dependent manner. UAP56 controls long circular RNA export, whereas URH49 controls short circular RNA export. Circular RNAs degradation is triggered by three endonuclease (AGO, RNase P, and RNase L), and promoted by an evolutionarily conserved factor-GW182.

\section{Degradation}

Different from most species of linear RNAs, circular RNAs are naturally resistant to exonuclease, and have long half-lives for their lack of 3' and 5' end. Depletion of several canonical RNA degradation factors had little effect on circular RNA levels, suggesting that circular RNA degradation seems to employ a regulatory pathway that is distinct from linear RNAs'. (i) AGO: An early study demonstrated that near perfect microRNA binding sites can trigger cleavage of some circular RNAs in an Ago-slicerdependent pathway (Figure 2) [18]. The most well-known example is ciRS7 (circular RNA sponge for miRNA-7) which has complementary target sites for miRNA-671. The interaction with miRNA-671 directs cleavage of ciRS-7 by Ago2 in human cells. (ii) GW182: Using RNAi screening strategy, our group identified an evolutionarily conserved factor (GW182 in Drosophila; TNRC6A, TNRC6B, and TNRC6C in human) controls circular RNA stability in a P-body or AGO independent manner recently (Figure 2). Surprisingly, the expression of parental linear RNAs were not affected upon GW182 depletion [19]. Although GW182's role in circular RNA stability control is yet to be identified, we hypothesize that GW182 might act as ae a molecular platform to recruit many decay factors, or GW182 might affect the subcellular localization of circular RNAs. (iii) RNase $\boldsymbol{P}$ 
and RNase L: $\mathrm{m}^{6} \mathrm{~A}$-modified circular RNAs have been shown to associates with YTHDF2 in an HRSP12-dependent manner and be cleaved by an endonuclease-RNase P selectively (Figure 2) [20]. Circular RNA degradation is also associated with innate immunity. RNase L, a widely expressed cytoplasmic endonuclease, can mediate circular RNA degradation genome-widely upon viral infection and PKR activation (Figure 2) [21].

\section{Molecular Functions}

Circular RNAs have diverse molecular functions in eukaryotic cells. (i) MircoRNA sponges: many studies have proved that plenty of exonic circular RNAs have multiple microRNA binding sites, and thereby can sequester microRNAs in the cytoplasm to control gene expression [22-28]. For example, circHIPK3 acts as a sponge to modulate cell growth and division by decreasing activities of multiple microRNAs [24]. "Circular RNA-microRNA-mRNA" axis serves as a new way of post-transcriptional regulation. (ii) Transcriptional regulation: RNA polymerase II associated exon-intron circular RNAs were found to control their parental mRNA production in the nucleus via RNA-RNA interaction with U1 snRNP [10]. Blocking the RNA-RNA interaction impairs the interactions of EIciRNAs with parental gene promoter and RNA polymerase II, thereby decreasing transcription activities of their parental mRNAs. (iii) Splicing regulation: A role of circular RNAs in splicing events has also been proved by the observation that circSEP3 is able to control SEP3 mRNA splicing and induce transcriptional pausing by forming a strong DNA-RNA hybrid with its cognate DNA locus in Arabidopsis [29]. (iv) Translation templates: Due to lack of both 5' caps and 3' ploy(A) tails, most circular RNA were first thought to be untranslatable. However, a small subset of natural circular RNAs were found as translation templates that can be used to produce proteins [30-32]. For example, a conserved and highly expressed circular RNA-circZNF609 contains an open reading frame and encodes a protein in a cap-independent manner, but the functions of these circular RNAencoded proteins are yet to be defined [30]. (v) Protein subcellular localization: two mitochondrial genome encoded circular RNAs (mecciRNAs) were reported to be involved in mitochondrial protein import via a polynucleotide phosphorylase (PNPASE), indicating that at least a subset of circular RNAs might affect protein subcellular localization [33]. However, the transportation and production of these mecciRNAs still remain unknown.

\section{CIRCULAR RNA IN BRAIN DISORDERS}

Most circular RNAs have been reported to be weekly expressed [13,14,34,35]; however, some circular RNAs are more abundant than their parental linear counterparts [36-39]. In Drosophila DL1 cells, circlaccase2 expression is ten times higher than linear laccase 2 mRNA, suggesting at least a subset of circular transcripts are major products of certain genes 
[3,11]. Surprisingly, comprehensive sequencing data demonstrated that there are thousands of circular transcripts accumulating in animal brains during aging and neuronal differentiation [30,35-37,40-44]. Following induction of neuronal activity and plasticity, the expression pattern of circular RNAs changes, while their parental linear counterparts are little affected. It will be interesting to explore the mechanism of such circularlinear transcripts balance regulation. Furthermore, some brain-enriched circular RNAs are evolutionarily conserved in expression and sequence from Drosophila to human [37,43]. Finally, there are much more circular RNAs in human brains than that in mouse brains. The probable reason is that these circular transcripts are not equally distributed in animal brains, but highly enriched in the synapses, and the synaptic density in human cerebral cortex is much higher than that in mouse [36,37]. Together, these data indicate that brain-enriched circular RNAs may have roles in brain functions.

\section{Circular RNA in Alzheimer's Disease}

Emerging studies have shown that circular RNAs are correlated with Alzheimer's disease (AD), which is a form of dementia and a neurodegenerative disease that progresses slowly over time among old people. ciRS-7 has been shown to be an endogenous mircoRNA sponge that sequester miRNA-7 via base pairing, thereby suppressing its functions and modulating its targets' translation [22]. ciRS-7 is dramatically downregulated in Alzheimer's disease affected brain cells compared to age-matched control hippocampal CA1, resulting in significantly accumulated miRNA-7 [45,46]. As one of miRNA-7's targets, UBE2A (ubiquitin conjugating enzyme E2A) is important in removal of amyloid peptides, and has been reported to be involved in several neurological diseases, such as AD [47,48]. These excess miRNA-7 thereby significantly reduce the translation of UBE2A mRNA. Subsequently, loss of UBE2A lead to accumulation of waste products, such as amyloid peptides and senile plaque, in AD brain cells. These data indicate that deficits in "circRNAmicroRNA-mRNA" system may present an important reason of widely observed change of gene expression in AD. Interestingly, alternative splicing patterns have been reported to change in $\mathrm{AD}$, and the expression of circular RNAs are significantly altered in AD-affected brain cells genome-widely [49-52]. For example, there 444 circular RNA were significantly upregulated, while 111 circular RNAs were downregulated in the hippocampus of $\mathrm{AD}$ rats compared to sham control groups [51]; RNA sequencing analysis of human brain astrocytes by Sekar et al. showed that there were over 2000 circular RNAs were uniquely expressed in AD cohort [52]. On the other hand, from Drosophila, C. elegans, mice to human, some circular RNAs are evolutionarily conserved, and there is obvious trend that the expression of many circular RNAs increases with age [42,43,53], suggesting that circular RNAs may participate in aging and AD 
pathogenesis, and represent a potential class of clinic biomarkers and therapeutic targets.

\section{Circular RNA in Neuropsychiatric Disorders}

As mentioned above, ciRS-7 is a conserved circular transcripts that is highly enriched in animal brains but lowly expressed in other organs. CiRS-7 has also been found to have partially complementary target sites for miRNA-7 and near perfect binding sites for miRNA-671, but whether ciRS-7 inhibits or protects miRNA-7 might depend on the cellular context [45,46,54-57]. Indeed, Piwecka et al. have beautifully demonstrated that ciRS-7 is important for sensorimotor gating and synaptic transmission [55]. To explore the roles of ciRS-7 in brain, loss-of-function mutant mice was generated by removal of ciRS-7 locus. Interestingly, miRNA-7 expression decreased, whereas miRNA-671 expression increased in ciRS-7 KO brain. It is important to note that these two microRNAs were unaffected in other tissues of ciRS-7 KO mice, suggesting a specific and posttranscriptional regulation of circular RNA-microRNA axis in brain. Subsequently, as targets of miRNA-7, immediate early genes that have roles in neuronal activities were significantly upregulated globally, thereby leading to dysfunction of excitatory synaptic transmission. Behavioral assays confirmed that mice with depletion of ciRS-7 showed deficits in prepulse inhibition of the startle reflex. Taken together, Piwecka et al. have proved that ciRS-7-microRNAs-target mRNAs axis is important in neuropsychiatric disorders [55]. However, a following study by Kleaveland et al. have clearly showed that the Cyrano long noncoding RNA can induce accumulation of ciRS-7 by reducing miRNA-7 levels. Without Cyrano, excess miRNA-7 causes cytoplasmic degradation of ciRS-7 in neurons, in part through enhanced slicing of ciRS-7 by miRNA-671. The results by Kleaveland et al. suggest a regulatory network formed by the Cyrano long noncoding RNA, ciRS-7, miRNA-7, and miRNA-671 [54].

\section{Circular RNA in Other Brain Disorders}

(i) Memory: PAIP2 has been reported as a pivotal translational regulator of synaptic plasticity and memory via poly(A)-binding protein (PABP) reactivation [58,59]. Nuclear circPAIP2 (exon-intron circular RNA) can regulate PAIP2 gene transcription in cis [10], thereby affecting memory development. (ii) Major depressive disorder: circular RNAs have also been shown to be correlated with major depressive disorder (MDD) [60,61]. Among circular RNAs with varying expression in MDD patients, only hsa_circRNA_103636 expression was significantly rescued after 8-week antidepressant regimens, suggesting that hsa_circRNA_103636 can be an ideal biomarker for the diagnosis of MDD [60]. (iii) Multiple sclerosis: current researches have demonstrated that the levels of several circular RNAs were significantly affected in multiple sclerosis cases. For example, Gasdermin B (GSDMB) circular RNA was found to be upregulated [62], whereas circ_0005402 and circ_0035560 
were reported to be underexpressed in multiple sclerosis patients [63]. Thus circular RNAs could be also used as biomarkers for the multiple sclerosis [62,63].

\section{PERSPECTIVES AND MOVING FORWARD: CIRCULAR RNAS AS PROMISING BIOMARKERS FOR HUMAN DISEASE}

As a special class of non-coding RNAs, circular RNAs have caught many researchers' attention, and triggered a number of exciting studies. Thousands of circular RNAs (including exonic, intronic, and exon-intron circular RNAs) have been identified across species by a variety of RNA-seq data, and some of them have been reported to impact normal and disease states, including cancer, cardiovascular disease, neurological disease, diabetes and so on [64-71].

Because (i) many circular RNAs have been clearly reported to be expressed in a tissue- or development stage-specific manner, (ii) the stable structure and natural nuclease-resistance are the common features of most circular RNAs, circular RNAs can serve as a novel class of biomarkers for diagnostics and a potential therapeutic targets. For example, lung cancer (LC) is one of the leading causes of death worldwide, but there is not an ideal blood biomarker for LC so far. Recent studies revealed that the levels of certain circular RNAs (e.g., has_circ_0013958, hsa_circ_0000064 and hsa_circRNA_103809) were drastically increased in lung cancer tissues. Depletion of these circular RNAs significantly suppressed lung cancer cell proliferation and invasion [72-74], indicating that they could be used for not only the novel targets in LC therapy but also the blood biomarkers in LC early detection.

But it is important to point out that there are still several issues need to be addressed prior to the application of circular RNA biomarkers in clinical therapy. (i) Reliability and sensitivity: because the clinical biomarker studies related to circular RNAs are still very limited, and the functions of most circular RNAs are still unknown, further experiments need to be employed to confirm the reliability and sensitivity of these potential biomarkers. (ii) Cost: A low-cost approach that is used to detect circular RNAs in blood cells or exosomes need to be explored, because detection of circular RNAs in these samples is very expensive and timeconsuming currently. In conclusion, circular RNA biology represents a novel and promising field in current medicine, biomarker and clinical researches.

\section{AUTHOR CONTRIBUTIONS}

$\mathrm{RJ}, \mathrm{ZL}$, and $\mathrm{CH}$ wrote the paper. RJ contributed to the figures in the paper.

\section{CONFLICTS OF INTEREST}

The authors declare that they have no conflicts of interest. 


\section{FUNDINGS}

This work was sponsored by Natural Science Foundation of Chongqing, China (cstc2019jcyj-msxmX0085 to $\mathrm{CH}$ ) and 100 Talent Program (Chongqing University, 0304001104433 to $\mathrm{CH}$ ).

\section{ACKNOWLEDGMENTS}

We thank Dr. Ge Shan and the members of Shan's laboratory (University of Science and Technology of China) for discussions.

\section{REFERENCES}

1. Liang D, Wilusz JE. Short intronic repeat sequences facilitate circular RNA production. Genes Dev. 2014;28(20):2233-47.

2. Zhang XO, Wang HB, Zhang Y, Lu X, Chen LL, Yang L. Complementary sequence-mediated exon circularization. Cell. 2014;159(1):134-47.

3. Kramer MC, Liang D, Tatomer DC, Gold B, March ZM, Cherry S, et al. Combinatorial control of Drosophila circular RNA expression by intronic repeats, hnRNPs, and SR proteins. Genes Dev. 2015;29(20):2168-82.

4. Conn SJ, Pillman KA, Toubia J, Conn VM, Salmanidis M, Phillips CA, et al. The RNA binding protein quaking regulates formation of circRNAs. Cell. 2015;160(6):1125-34.

5. Errichelli L, Dini Modigliani S, Laneve P, Colantoni A, Legnini I, Capauto D, et al. FUS affects circular RNA expression in murine embryonic stem cellderived motor neurons. Nat Commun. 2017;8:14741.

6. Ivanov A, Memczak S, Wyler E, Torti F, Porath HT, Orejuela MR, et al. Analysis of intron sequences reveals hallmarks of circular RNA biogenesis in animals. Cell Rep. 2015;10(2):170-7.

7. Ashwal-Fluss R, Meyer M, Pamudurti NR, Ivanov A, Bartok O, Hanan M, et al. circRNA biogenesis competes with pre-mRNA splicing. Mol Cell. 2014;56(1): 55-66.

8. Barrett SP, Wang PL, Salzman J. Circular RNA biogenesis can proceed through an exon-containing lariat precursor. Elife. 2015;4:e07540.

9. Kelly S, Greenman C, Cook PR, Papantonis A. Exon Skipping Is Correlated with Exon Circularization. J Mol Biol. 2015;427(15):2414-7.

10. Li Z, Huang C, Bao C, Chen L, Lin M, Wang X, et al. Exon-intron circular RNAs regulate transcription in the nucleus. Nat Struct Mol Biol. 2015;22(3):256-64.

11. Liang D, Tatomer DC, Luo Z, Wu H, Yang L, Chen LL, et al. The Output of Protein-Coding Genes Shifts to Circular RNAs When the Pre-mRNA Processing Machinery Is Limiting. Mol Cell. 2017;68(5):940-54.e3.

12. Zhang Y, Zhang XO, Chen T, Xiang JF, Yin QF, Xing YH, et al. Circular intronic long noncoding RNAs. Mol Cell. 2013;51(6):792-806.

13. Salzman J, Gawad C, Wang PL, Lacayo N, Brown PO. Circular RNAs are the predominant transcript isoform from hundreds of human genes in diverse cell types. PLoS One. 2012;7(2):e30733. 
14. Jeck WR, Sorrentino JA, Wang K, Slevin MK, Burd CE, Liu J, et al. Circular RNAs are abundant, conserved, and associated with ALU repeats. RNA. 2013;19(2):141-57.

15. Huang C, Liang D, Tatomer DC, Wilusz JE. A length-dependent evolutionarily conserved pathway controls nuclear export of circular RNAs. Genes Dev. 2018;32(9-10):639-44.

16. Li Z, Kearse MG, Huang C. The nuclear export of circular RNAs is primarily defined by their length. RNA Biol. 2019;16(1):1-4.

17. Wan Y, Hopper AK. Size matters: conserved proteins function in lengthdependent nuclear export of circular RNAs. Genes Dev. 2018;32(9-10):600-1.

18. Hansen TB, Wiklund ED, Bramsen JB, Villadsen SB, Statham AL, Clark SJ, et al. miRNA-dependent gene silencing involving Ago2-mediated cleavage of a circular antisense RNA. EMBO J. 2011;30(21):4414-22.

19. Jia R, Xiao MS, Li Z, Shan G, Huang C. Defining an evolutionarily conserved role of GW182 in circular RNA degradation. Cell Discov. 2019;5:45.

20. Park OH, Ha H, Lee Y, Boo SH, Kwon DH, Song HK, et al. Endoribonucleolytic Cleavage of m(6)A-Containing RNAs by RNase P/MRP Complex. Mol Cell. 2019;74(3):494-507.e8.

21. Liu CX, Li X, Nan F, Jiang S, Gao X, Guo SK, et al. Structure and Degradation of Circular RNAs Regulate PKR Activation in Innate Immunity. Cell. 2019;177(4):865-80.e21.

22. Hansen TB, Jensen TI, Clausen BH, Bramsen JB, Finsen B, Damgaard CK, et al. Natural RNA circles function as efficient microRNA sponges. Nature. 2013;495(7441):384-8.

23. Memczak S, Jens M, Elefsinioti A, Torti F, Krueger J, Rybak A, et al. Circular RNAs are a large class of animal RNAs with regulatory potency. Nature. 2013;495(7441):333-8.

24. Zheng Q, Bao C, Guo W, Li S, Chen J, Chen B, et al. Circular RNA profiling reveals an abundant circHIPK3 that regulates cell growth by sponging multiple miRNAs. Nat Commun. 2016;7:11215.

25. Thomson DW, Dinger ME. Endogenous microRNA sponges: evidence and controversy. Nat Rev Genet. 2016;17(5):272-83.

26. Han D, Li J, Wang H, Su X, Hou J, Gu Y, et al. Circular RNA circMTO1 acts as the sponge of microRNA-9 to suppress hepatocellular carcinoma progression. Hepatology. 2017;66(4):1151-64.

27. Chen J, Li Y, Zheng Q, Bao C, He J, Chen B, et al. Circular RNA profile identifies circPVT1 as a proliferative factor and prognostic marker in gastric cancer. Cancer Lett. 2017;388:208-19.

28. He Q, Zhao L, Liu Y, Liu X, Zheng J, Yu H, et al. circ-SHKBP1 Regulates the Angiogenesis of U87 Glioma-Exposed Endothelial Cells through miR544a/FOXP1 and miR-379/FOXP2 Pathways. Mol Ther Nucleic Acids. 2018;10:331-48.

29. Conn VM, Hugouvieux V, Nayak A, Conos SA, Capovilla G, Cildir G, et al. A circRNA from SEPALLATA3 regulates splicing of its cognate mRNA through Rloop formation. Nat Plants. 2017;3:17053. 
30. Legnini I, Di Timoteo G, Rossi F, Morlando M, Briganti F, Sthandier O, et al. Circ-ZNF609 Is a Circular RNA that Can Be Translated and Functions in Myogenesis. Mol Cell. 2017;66(1):22-37.e9.

31. Pamudurti NR, Bartok O, Jens M, Ashwal-Fluss R, Stottmeister C, Ruhe L, et al. Translation of CircRNAs. Mol Cell. 2017;66(1):9-21.e7.

32. Yang Y, Fan X, Mao M, Song X, Wu P, Zhang Y, et al. Extensive translation of circular RNAs driven by N(6)-methyladenosine. Cell Res. 2017;27(5):626-41.

33. Liu X, Wang X, Li J, Hu S, Deng Y, Yin H, et al. The identification of mecciRNAs and their roles in mitochondrial entry of proteins. bioRxiv 668665 [Preprint]. 2019 Jun 12. doi: 10.1101/668665

34. Guo JU, Agarwal V, Guo H, Bartel DP. Expanded identification and characterization of mammalian circular RNAs. Genome Biol. 2014;15(7):409.

35. Zhang Y, Xue W, Li X, Zhang J, Chen S, Zhang JL, et al. The Biogenesis of Nascent Circular RNAs. Cell Rep. 2016;15(3):611-24.

36. You X, Vlatkovic I, Babic A, Will T, Epstein I, Tushev G, et al. Neural circular RNAs are derived from synaptic genes and regulated by development and plasticity. Nat Neurosci. 2015;18(4):603-10.

37. Rybak-Wolf A, Stottmeister C, Glazar P, Jens M, Pino N, Giusti S, et al. Circular RNAs in the Mammalian Brain Are Highly Abundant, Conserved, and Dynamically Expressed. Mol Cell. 2015;58(5):870-85.

38. Zhang XO, Dong R, Zhang Y, Zhang JL, Luo Z, Zhang J, et al. Diverse alternative back-splicing and alternative splicing landscape of circular RNAs. Genome Res. 2016;26(9):1277-87.

39. Salzman J, Chen RE, Olsen MN, Wang PL, Brown PO. Cell-type specific features of circular RNA expression. PLoS Genet. 2013;9(9):e1003777.

40. Chen BJ, Huang S, Janitz M. Changes in circular RNA expression patterns during human foetal brain development. Genomics. 2019;111(4):753-8.

41. Veno MT, Hansen TB, Veno ST, Clausen BH, Grebing M, Finsen B, et al. Spatiotemporal regulation of circular RNA expression during porcine embryonic brain development. Genome Biol. 2015;16:245.

42. Gruner H, Cortes-Lopez M, Cooper DA, Bauer M, Miura P. CircRNA accumulation in the aging mouse brain. Sci Rep. 2016;6:38907.

43. Westholm JO, Miura P, Olson S, Shenker S, Joseph B, Sanfilippo P, et al. Genome-wide analysis of drosophila circular RNAs reveals their structural and sequence properties and age-dependent neural accumulation. Cell Rep. 2014;9(5):1966-80.

44. Szabo L, Morey R, Palpant NJ, Wang PL, Afari N, Jiang C, et al. Statistically based splicing detection reveals neural enrichment and tissue-specific induction of circular RNA during human fetal development. Genome Biol. 2015;16:126.

45. Zhao Y, Alexandrov PN, Jaber V, Lukiw WJ. Deficiency in the Ubiquitin Conjugating Enzyme UBE2A in Alzheimer's Disease (AD) is Linked to Deficits in a Natural Circular miRNA-7 Sponge (circRNA; ciRS-7). Genes (Basel). 2016;7(12):116.

46. Lukiw WJ. Circular RNA (circRNA) in Alzheimer's disease (AD). Front Genet. 2013;4:307. 
47. Bingol B, Sheng M. Deconstruction for reconstruction: the role of proteolysis in neural plasticity and disease. Neuron. 2011;69(1):22-32.

48. Lonskaya I, Shekoyan AR, Hebron ML, Desforges N, Algarzae NK, Moussa CE. Diminished parkin solubility and co-localization with intraneuronal amyloidbeta are associated with autophagic defects in Alzheimer's disease. J Alzheimers Dis. 2013;33(1):231-47.

49. Huang JL, Qin MC, Zhou Y, Xu ZH, Yang SM, Zhang F, et al. Comprehensive analysis of differentially expressed profiles of Alzheimer's disease associated circular RNAs in an Alzheimer's disease mouse model. Aging. 2018;10(2): 253-65.

50. Huang JL, Xu ZH, Yang SM, Yu C, Zhang F, Qin MC, et al. Identification of Differentially Expressed Profiles of Alzheimer's Disease Associated Circular RNAs in a Panax Notoginseng Saponins-Treated Alzheimer's Disease Mouse Model. Comput Struct Biotechnol J. 2018;16:523-31.

51. Wang Z, Xu P, Chen B, Zhang Z, Zhang C, Zhan Q, et al. Identifying circRNAassociated-ceRNA networks in the hippocampus of Abeta1-42-induced Alzheimer's disease-like rats using microarray analysis. Aging (Albany NY). 2018;10(4):775-88.

52. Sekar S, Cuyugan L, Adkins J, Geiger P, Liang WS. Circular RNA expression and regulatory network prediction in posterior cingulate astrocytes in elderly subjects. BMC Genomics. 2018;19(1):340.

53. Rodriguez SA, Grochova D, McKenna T, Borate B, Trivedi NS, Erdos MR, et al. Global genome splicing analysis reveals an increased number of alternatively spliced genes with aging. Aging Cell. 2016;15(2):267-78.

54. Kleaveland B, Shi CY, Stefano J, Bartel DP. A Network of Noncoding Regulatory RNAs Acts in the Mammalian Brain. Cell. 2018;174(2):350-62.e17.

55. Piwecka M, Glazar P, Hernandez-Miranda LR, Memczak S, Wolf SA, RybakWolf A, et al. Loss of a mammalian circular RNA locus causes miRNA deregulation and affects brain function. Science. 2017;357(6357):eaam8526.

56. Weng W, Wei Q, Toden S, Yoshida K, Nagasaka T, Fujiwara T, et al. Circular RNA ciRS-7-A Promising Prognostic Biomarker and a Potential Therapeutic Target in Colorectal Cancer. Clin Cancer Res. 2017;23(14):3918-28.

57. Yu L, Gong X, Sun L, Zhou Q, Lu B, Zhu L. The Circular RNA Cdr1as Act as an Oncogene in Hepatocellular Carcinoma through Targeting miR-7 Expression. PLoS One. 2016;11(7):e0158347.

58. Khoutorsky A, Yanagiya A, Gkogkas CG, Fabian MR, Prager-Khoutorsky M, Cao $\mathrm{R}$, et al. Control of synaptic plasticity and memory via suppression of poly(A)binding protein. Neuron. 2013;78(2):298-311.

59. Bonin RP, De Koninck Y. Reconsolidation and the regulation of plasticity: moving beyond memory. Trends Neurosci. 2015;38(6):336-44.

60. Cui X, Niu W, Kong L, He M, Jiang K, Chen S, et al. hsa_circRNA_103636: potential novel diagnostic and therapeutic biomarker in Major depressive disorder. Biomark Med. 2016;10(9):943-52.

61. Jiang G, Ma Y, An T, Pan Y, Mo F, Zhao D, et al. Relationships of circular RNA with diabetes and depression. Sci Rep. 2017;7(1):7285. 
62. Cardamone G, Paraboschi EM, Rimoldi V, Duga S, Solda G, Asselta R. The Characterization of GSDMB Splicing and Backsplicing Profiles Identifies Novel Isoforms and a Circular RNA That Are Dysregulated in Multiple Sclerosis. Int J Mol Sci. 2017;18(3):576.

63. Iparraguirre L, Munoz-Culla M, Prada-Luengo I, Castillo-Trivino T, Olascoaga J, Otaegui D. Circular RNA profiling reveals that circular RNAs from ANXA2 can be used as new biomarkers for multiple sclerosis. Hum Mol Genet. 2017;26(18):3564-72.

64. Patop IL, Kadener S. circRNAs in Cancer. Curr Opin Genet Dev. 2018;48:121-7.

65. Wilusz JE. A 360 degrees view of circular RNAs: From biogenesis to functions. Wiley Interdiscip Rev RNA. 2018;9(4):e1478.

66. Haque S, Harries LW. Circular RNAs (circRNAs) in Health and Disease. Genes (Basel). 2017;8(12):353.

67. Greene J, Baird AM, Brady L, Lim M, Gray SG, McDermott R, et al. Circular RNAs: Biogenesis, Function and Role in Human Diseases. Front Mol Biosci. 2017;4:38.

68. Kristensen LS, Andersen MS, Stagsted LVW, Ebbesen KK, Hansen TB, Kjems J. The biogenesis, biology and characterization of circular RNAs. Nat Rev Genet. 2019;20(11):675-91.

69. Li X, Yang L, Chen LL. The Biogenesis, Functions, and Challenges of Circular RNAs. Mol Cell. 2018;71(3):428-42.

70. Wilusz JE. Circular RNAs: Unexpected outputs of many protein-coding genes. RNA Biol. 2017;14(8):1007-17.

71. Zhang Z, Yang T, Xiao J. Circular RNAs: Promising Biomarkers for Human Diseases. EBioMedicine. 2018;34:267-74.

72. Liu W, Ma W, Yuan Y, Zhang Y, Sun S. Circular RNA hsa_circRNA_103809 promotes lung cancer progression via facilitating ZNF121-dependent MYC expression by sequestering miR-4302. Biochem Biophys Res Commun. 2018;500(4):846-51.

73. Zhu X, Wang X, Wei S, Chen Y, Chen Y, Fan X, et al. hsa_circ_0013958: a circular RNA and potential novel biomarker for lung adenocarcinoma. FEBS J. 2017;284(14):2170-82.

74. Luo YH, Zhu XZ, Huang KW, Zhang Q, Fan YX, Yan PW, et al. Emerging roles of circular RNA hsa_circ_0000064 in the proliferation and metastasis of lung cancer. Biomed Pharmacother. 2017;96:892-8.

How to cite this article:

Jia R, Li Z, Huang C. Circular RNAs in Brain Disorders. Med One. 2019;4:e190025. https://doi.org/ $\underline{10.20900 / \mathrm{mo} 0.20190025}$ 